\title{
Microbial succession during wheat bran fermentation and colonisation by human faecal microbiota as a result of niche diversification
}

\author{
Kim De Paepe $\mathbb{D}^{1} \cdot$ Joran Verspreet $\mathbb{D}^{2} \cdot$ Christophe M. Courtin $\mathbb{D}^{2} \cdot$ Tom Van de Wiele $^{1}$
}

Received: 30 August 2018 / Revised: 27 June 2019 / Accepted: 29 September 2019 / Published online: 11 November 2019

(c) The Author(s) 2019. This article is published with open access

\begin{abstract}
The human gut can be viewed as a flow-through system with a short residence time, a high turnover rate and a spatial gradient of physiological conditions. As a consequence, the gut microbiota is exposed to highly fluctuating environmental determinants presented by the host and diet. Here, we assessed the fermentation and colonisation of insoluble wheat bran by faecal microbiota of three individuals at an unprecedented sampling intensity. Time-resolved 16S rRNA gene amplicon sequencing, revealed a dynamic microbial community, characterised by abrupt shifts in composition, delimiting states with a more constant community, giving rise to a succession of bacterial taxa alternately dominating the community over a $72 \mathrm{~h}$ timespan. Early stages were dominated by Enterobacteriaceae and Fusobacterium species, growing on the carbohydratelow, protein rich medium to which wheat bran was supplemented. The onset of wheat bran fermentation, marked by a spike in short chain fatty acid production with an increasing butyrate proportion and an increased endo-1,4- $\beta$-xylanase activity, corresponded to donor-dependent proportional increases of Bacteroides ovatus/stercoris, Prevotella copri and Firmicutes species, which were strongly enriched in the bran-attached community. Literature and database searches provided novel insights into the metabolic and growth characteristics underlying the observed succession and colonisation, illustrating the potency of a time-resolved analysis to increase our understanding of gut microbiota dynamics upon dietary modulations.
\end{abstract}

\section{Introduction}

The human gut harbours an indigenous microbial community characterised by a tremendous inter-individual variability, comprising around 100-160 different species within a single individual [1-6]. Most of the research initiatives directed towards the characterisation of this community provide snapshots of the faecal microbiota composition at a single point in time and the knowledge gap on intra-

Supplementary information The online version of this article (https:// doi.org/10.1038/s41396-019-0550-5) contains supplementary material, which is available to authorised users.

Tom Van de Wiele

Tom.VandeWiele@UGent.be

1 Department of Biotechnology, Faculty of Bioscience Engineering, Center for Microbial Ecology and Technology (CMET), Ghent University, Ghent, Belgium

2 Laboratory of Food Chemistry and Biochemistry, Leuven Food Science and Nutrition Research Centre (LFoRCe), Faculty of Bioscience Engineering, KU Leuven, Heverlee, Belgium individual variability in microbiome composition is immense [7-9]. Longitudinal studies on the dynamics of the faecal microbiome from human individuals are sparse and often only include a limited set of time points [10].

The temporal variation in the gut microbiota composition is often studied early in life. The largest shifts occur during the first 3-4 years, when the gut microbial community establishes [11-13]. In terms of species absence/presence, the adult microbial community within one individual is relatively stable over time [2,14-16]. The proportions of microbial taxa, however, are more subjected to temporal changes, reflecting a high responsiveness to perturbations, such as diarrhoeal episodes, distant travelling, antibiotic therapy and changing host conditions upon ageing [9, 14, 16-19]. One of the most influential and extensively studied forces shaping the human gut microbiota is the diet [14, 20-23]. With the exception of a few studies, the dietary impact on gut microbiota composition in human intervention trials is only assessed on the long-term and restricted to baseline and endpoint measurements [23-30].

Nevertheless, there are intriguing reports on the shortterm responsiveness of the microbiome towards dietary 
transitions. Analysing faecal samples from an intervention study twice a week, Walker et al. [31] revealed a rapid response within 3-4 days. This timeframe was further narrowed in a study by David et al. [14] showing that a high fibre intake correlated with next-day enrichment of Bifidobacteria, Roseburia and Eubacterium rectale. These in vivo findings were confirmed in vitro by Aguirre et al. [32]. Obviously, substrate fermentation in the gut is a short-term process, confined by intestinal transit time, which can vary between individuals, but is in the order of 18 up to $60 \mathrm{~h}$ [33]. During gastrointestinal transit of a dietary substituent an ecological succession of primary colonisers, primary degraders, and cross-feeding microorganisms takes place, shaping the gut microbiome with every meal. This may have a long-term impact on microbiome composition and activity and eventually also the human host. Capturing the short-term dynamics of microbial succession can thus provide us with a better understanding of a fundamental process that underlies human gut microbiome composition. However, monitoring microbial dynamics requires highly frequent sampling. Despite the physiological relevance of human in vivo trials, frequent sampling of faecal microbiota or inaccessible gut regions is impossible. In vitro models allow frequent and regular sampling without ethical constraints [34], while the reduced complexity permits the study of specific dietary compounds at a high level of control, abstracting possible confounding effects due to the background diet or participant non-adherence [35].

In this study, we examined the time course of wheat bran colonisation and fermentation. The choice for wheat bran was made for its high dietary fibre content and for its recalcitrance to digestion in the upper gastrointestinal tract, making it available as a fermentation substrate for gut bacteria [31, 36-39]. Due to its insoluble nature and chemical complexity, wheat bran degradation requires the concerted action of a multitude of enzymes and involves a range of gut bacteria, which might act simultaneously or sequentially [36, 40-43]. We monitored the succession of gut microbiota members during wheat bran fermentation with time-resolved next-generation 16S rRNA gene amplicon sequencing. Both the luminal and the wheat branattached microbial populations were characterised and interindividual differences were considered with separate incubations from three donors, selected from a preliminary screening experiment [40].

\section{Materials and methods}

\section{Experimental set-up}

Commercial wheat bran from the harvest of 2014 was supplied by Dossche Mills (Deinze, Belgium) and chemically characterised as described by De Paepe et al. [44] (Table S1). Upper digestive tract passage of wheat bran was mimicked through an in vitro batch digestion, adapted from Minekus et al. [45], as described by De Paepe et al. [40].

Subsequently, wheat bran was separately incubated with the human faecal microbiota derived from three healthy individuals, without a history of antibiotic use 6 months prior to the study. All three donors consumed a mixed Western diet. Donors were selected based on a divergent response to wheat bran in a previous experiment [40]. The donors differed in Bacteroides (donor 1), Lachnospiraceae (donor 2) and Prevotella (donor 3) signature and the nature of the produced SCFA. Donors 2 and 3 were characterised by a higher butyrate ratio in response to wheat bran, whereas in donor 1 propionate prevailed. The stool samples from donors 1 and 2 were sausage shaped (corresponding to Bristol Stool Score type3), while donor 3 produced a looser stool (corresponding to Bristol Stool Score type5). It was previously shown that in vitro incubation of the faecal sample for $48 \mathrm{~h}$ in a carbohydrate-low control medium induced shifts in the community but preserved richness and diversity [40]. Incubation research with faecal microbiota from human origin was approved by the ethical committee of the Ghent University hospital under registration number B670201214538. Faecal slurries were prepared as described by De Paepe et al. [40] and inoculated to obtain a final concentration of $2 \%$ faecal material $(\mathrm{w} / \mathrm{v})$ in penicillin flasks containing a carbohydrate-low medium supplemented with $1 \%$ wheat bran $(\mathrm{w} / \mathrm{v})$. The carbohydrate-low medium contained $3 \mathrm{gL}^{-1}$ yeast extract (Oxoid Ltd, Basingstoke, Hampshire, UK), $1 \mathrm{~g} \mathrm{~L}^{-1}$ peptone (Oxoid Ltd, Basingstoke, Hampshire, UK) and $1 \mathrm{~g} \mathrm{~L}^{-1}$ mucin from porcine stomach Type II (Sigma Aldrich, St. Louis, MO, US), dissolved in $0.1 \mathrm{M}$ phosphate buffer at $\mathrm{pH} 6.8$ [40]. A control without wheat bran was taken along for donors 2 and 3. The penicillin flasks were flushed with $\mathrm{N}_{2} / \mathrm{CO}_{2}$ during 30 cycles to generate anaerobic conditions and set at atmospheric pressure before incubation at $37^{\circ} \mathrm{C}$ and $90 \mathrm{rpm}$ on an orbital shaker (KS 4000 i control, IKA, Staufen, Germany).

The course of fermentation and wheat bran colonisation was followed up over time. Samples were taken $1 \mathrm{~h}$ after inoculation, in 2-h time intervals the first $24 \mathrm{~h}$ after inoculation and after 48 and $72 \mathrm{~h}$. For each time point and each donor, a separate tube was incubated in duplicate (further referred to as a biological replicate), as shown in Figure S1. At the foreseen sampling time point, the gas pressure and $\mathrm{pH}$ were measured. The unfermented wheat bran residue was collected by centrifugation for $5 \mathrm{~min}$ at $700 \times g$ and stored in $0.1 \mathrm{~g}$ aliquots at $-20^{\circ} \mathrm{C}$ for DNA extraction, after washing with phosphate buffer to remove nonattached and loosely attached microorganisms, as described by De Paepe et al. [40]. Liquid samples ('luminal samples') were aliquoted for 
DNA extraction (pellet obtained after centrifuging $250 \mu \mathrm{L}$ at $5000 \times g$ for $10 \mathrm{~min}$ ), enzyme activity and short chain fatty acid (SCFA) analysis after storage at $-20^{\circ} \mathrm{C}$ (Fig. S1). For the enzyme activity measurement, $2 \mathrm{~mL}$ sample was centrifuged for $10 \mathrm{~min}$ at $8000 \times g$. The supernatant, representing the extracellular enzyme fraction, was stored at $-20^{\circ} \mathrm{C}$. The pellet was washed twice with $2 \mathrm{~mL}$ wash buffer (phosphate

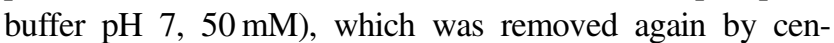
trifugation at $8000 \times g$ for $10 \mathrm{~min}$. The pellet was stored at $-20^{\circ} \mathrm{C}$. Prior to analysis $1.5 \mathrm{~mL}$ phosphate buffer $(\mathrm{pH} 7$, $50 \mathrm{mM})$ and $200 \mathrm{mg}$ glass beads $(0.11 \mathrm{~mm}$ Sartorius, Göttingen, Germany) were added to the pellet. Cell extracts, containing the membrane-associated and intracellular enzyme fraction, were obtained through mechanical lysis by multidirectional beating for two times $40 \mathrm{~s}$ at $1600 \mathrm{rpm}$ in a FastPrep-96 instrument (MP Biomedicals, Santa Ana, CA). $\alpha$-L-arabinofuranosidase and $\beta$-xylosidase activity were determined spectrophotometrically, using 4-nitrophenyl- $\beta$-Dxylopyranoside and 4-nitrophenyl- $\alpha$-L-arabinofuranoside as artificial substrates (Sigma Aldrich, St. Louis, MO, US). $2.5 \mathrm{mM}$ stock solutions of these substrates were freshly prepared in phosphate buffer $(\mathrm{pH} 7,50 \mathrm{mM})$. One hundred $\mu \mathrm{L}$ aliquots of these stock solutions were incubated in a 96-well plate with $100 \mu \mathrm{L}$ sample (1:10 diluted in phosphate buffer $\mathrm{pH} 7,50 \mathrm{mM})$. The enzymatic release of 4-nitrophenol was measured every $30 \mathrm{~min}$ for a period of $24 \mathrm{~h}$ at $405 \mathrm{~nm}, 37^{\circ} \mathrm{C}$ with an Infinite 200Pro plate reader (Tecan, Männedorf, Switzerland). Enzyme activities were expressed as the concentration 4-nitrophenol $(\mu \mathrm{M})$, inferred from the OD by reference to a 4-nitrophenol standard curve, released per minute in the linear range (up to $4 \mathrm{~h}$ ) at $\mathrm{pH} 7$ and $37^{\circ} \mathrm{C}$. The endo-1,4- $\beta$-xylanase enzyme activity was determined using the Xylazyme-AX assay (Megazyme, Bray, Ireland). Two hundred $\mu \mathrm{L}$ sample was diluted 2.5 times in McIlvaine buffer $\mathrm{pH} 6.8$ and incubated for $4 \mathrm{~h}$ at $37^{\circ} \mathrm{C}$ with a Xylazyme-AX tablet. Enzyme activities were expressed as the change in extinction values $\mathrm{h}^{-1} \mathrm{~mL}^{-1}$ sample measured at $590 \mathrm{~nm}$ (E590) compared to a blank without tablet, corrected by subtracting the E590 values of the buffer without sample. SCFA levels were measured using capillary gas chromatography coupled to a flame ionisation detector (Shimadzu, Hertogenbosch, the Netherlands) after diethyl ether extraction, as described by De Paepe et al. [40]. Samples were twofold diluted with milli-Q water.

\section{Microbial community analysis}

The microbial community was analysed using Illumina next-generation $16 \mathrm{~S}$ rRNA gene amplicon sequencing. Both the luminal suspension and the washed bran residue were analysed for the time points 1, 2, 4, 6, 8, 10, 12, 16, 20, 24, 48 and $72 \mathrm{~h}$ (Fig. S1). Biological replicates were only analysed from time points 2,10 and $48 \mathrm{~h}$.
DNA was extracted and DNA quality verified as described by De Paepe et al. [40]. Samples were sent out to LGC Genomics (Teddington, Middlesex, UK) for library preparation and sequencing on an Illumina Miseq platform. To assess sequencing quality, a mock community sample, with a composition as listed by De Paepe et al. [40] was included in triplicate.

The V3-V4 region of the 16S rRNA gene was amplified by PCR using primers derived from Klindworth et al. [46], with a slight modification to the reverse primer, $785 \mathrm{R}$ (5'-GACTACHVGGGTATCTAAKCC-3'), by introducing another degenerated position $(\mathrm{K})$ to make it more universal. The library preparation was performed, as described by De Paepe et al. [40]. The sequence data have been submitted to the NCBI (National Center for Biotechnology Information) database under accession number SRP127353.

\section{Bio-informatics and statistical analysis}

The mothur software package (v.1.39.5) and guidelines were used to process the amplicon data, as outlined in the Supplementary information [47]. The OTU table with taxonomy assignment according to RDP version 16 was loaded into R, version 3.4 .1 (2017-06-30) [48]. Singletons were removed [49]. For the most abundant OTUs the sequences retrieved from the $3 \%$ dissimilarity level fasta file, obtained in mothur, were classified using the RDP SeqMatch tool, restricting the database search to type strains with only near-full-length, good quality sequences and blasted in NCBI against the 16S rRNA gene sequences, selecting only type material, with optimisation of the BLAST algorithm for highly similar sequences (accession date: September 2017) [50-52]. Although identification to the species level based on short $300 \mathrm{bp}$ reads can be equivocal, the most likely species classification of some interesting OTUs is reported in the results and discussion section. In the event of inconsistencies in the results of the RDP SeqMatch tool and NCBI BLAST, no species level classification is provided. The results for the most abundant OTUs are shown in Table S2.

Statistical analysis was performed in $\mathrm{R}$, version 3.4.1 ( $\mathrm{R}$ [48]). Details regarding the applied ordination and clustering methods, network inference tools and formal hypothesis tests are provided in Supplementary Information.

\section{Results}

Wheat bran colonisation and fermentation by the faecal microbiota from three healthy individuals, selected based on their diverging response to wheat bran, were followed up through time by means of SCFA and enzyme activity 

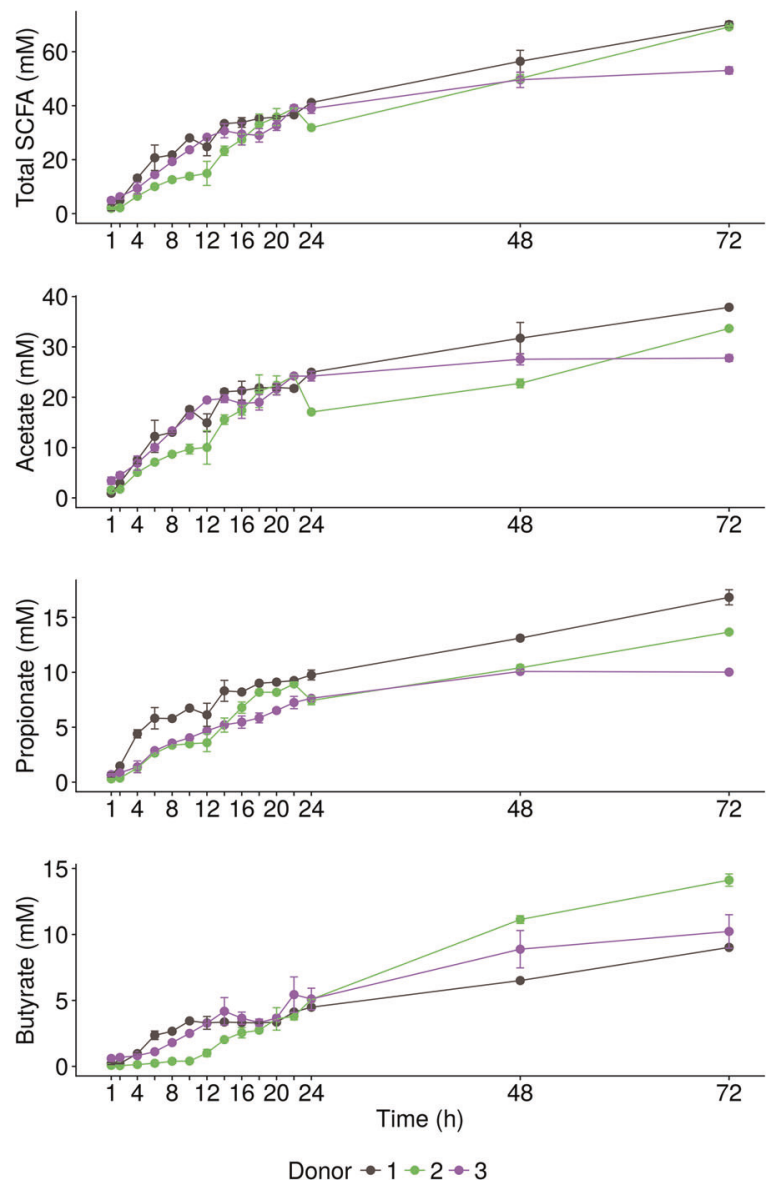

Fig. 1 SCFA production ( $n=2$ biological replicates) resulting from the incubation of wheat bran in the presence of a carbohydrate-low medium with the faecal material of three different donors for up to $72 \mathrm{~h}$

analysis and 16S rRNA gene amplicon sequencing of the luminal and bran-attached microbial community (Fig. S1).

The three donors fermented wheat bran, present in a carbohydrate-low medium, at a different rate and to a different extent (Fig. 1). In donor 2, fermentation proceeded in two phases, reaching a plateau after the first $12 \mathrm{~h}$, marking the depletion of components from the carbohydrate-low medium and the onset of wheat bran fermentation (Fig. 1, S2). In donors 1 and 3, the SCFA production increased more rapidly but levelled off $12-20 \mathrm{~h}$ after inoculation (Fig. 1). A comparison with the carbohydrate-low medium (Control) in donor 3 showed that wheat bran fermentation already kicked-off after $6 \mathrm{~h}$ but became more efficient when the carbohydrate-low medium was depleted after $20 \mathrm{~h}$ (Fig. S2). While donor 2 fermented wheat bran initially at a slower rate, after $72 \mathrm{~h}$, the total SCFA levels produced by donor $2(69.2 \pm 0.6 \mathrm{mM})$ were similar to donor $1(70.1 \pm$ $1.2 \mathrm{mM})$ and higher compared to donor $3(53.1 \pm 1.3 \mathrm{mM})$ (Fig. 1). Acetate production most rapidly increased after inoculation, followed by propionate production. Butyrate production was delayed with a lag phase of 4 up to $10 \mathrm{~h}$

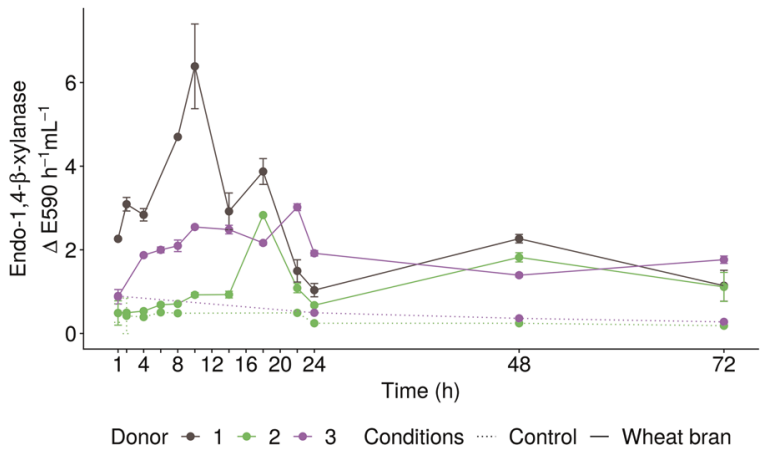

Fig. 2 Endo-1,4- $\beta$-xylanase enzyme activity $(n=2$ biological replicates) measured in the membrane-associated and intracellular fraction of liquid samples, consisting of a carbohydrate-low medium incubated with the faecal material of three different donors for up to $72 \mathrm{~h}$ in the absence (dotted line, Control) or presence (solid line, wheat bran) of wheat bran. Enzyme activity was expressed as the increase in extinction values measured at $590 \mathrm{~nm}$ (E590) per hour of incubation and per mililitre of sample

depending on the donor, resulting in an increasing butyrate to propionate ratio over time (Fig. S3). In donors 2 and 3, butyrate production approached or exceeded the propionate production after $48 \mathrm{~h}$. In donor 1 , on the other hand, propionate production was higher than butyrate at all time points. Branched SCFA production indicated that donor 3 displayed the lowest proteolytic activity (Fig. S4).

The slow donor-dependent fermentation of wheat bran was reflected in a donor-dependent increase of the endo-1,4$\beta$-xylanase activity, required for the primary degradation of wheat bran arabinoxylans, in the combined intracellular and membrane-associated enzyme fraction over time (Fig. 2). The slow onset of wheat bran fermentation in donor 2 corresponded to an initially lower enzyme activity compared to donors 1 and 3 . After $14 \mathrm{~h}$, however, a sudden peak in endo-1,4- $\beta$-xylanase activity was observed, paralleling the increment in SCFA production (Figs. 1, 2 and S2). A similar peak was observed in donor 1 after $8-10 \mathrm{~h}$, whereas in donor 3 a more gradual increase occurred with a smaller peak after $20 \mathrm{~h}$, coinciding with a further rise in SCFA production compared to the control (Fig. 2 and S2). The constant and low endo-1,4- $\beta$-xylanase activity levels (E590 $<0.2$ after $72 \mathrm{~h}$ ) in this control (Fig. 2) indicate that endo$1,4-\beta$-xylanase activity depended on the presence of wheat bran and the relation with the SCFA production illustrates that endo-1,4- $\beta$-xylanase is essential for the fermentation of wheat bran. Besides xylanase, other enzymes including $\beta$ xylosidase and $\alpha$-L-arabinofuranosidases are involved in the further depolymerisation of the substrate to fermentable xylose and arabinose monomers. These enzymes displayed a more variable activity, which was also present in the control (Fig. S5), indicating a less tight regulation of their activity by the wheat bran substrate. The extracellular enzyme fraction was not influenced by wheat bran 
supplementation. The extracellular enzyme activity was negligible after $72 \mathrm{~h}($ E590 $<0.2$ and a p-nitrophenol production $<0.06 \mu \mathrm{M} \mathrm{min}^{-1}$ ) compared to the intracellular and membrane-associated fractions for all enzymes and followed a similar decreasing pattern over time in the control and wheat bran incubations, indicating that this activity originated from enzymes released through cell disintegration in the faecal samples, exhibiting the highest extracellular activity (E590 $>3$ and p-nitrophenol production $>4$ $\mu \mathrm{M})$. Considering this low extracellular enzyme activity and given the required extracellular action of endo- $1,4-\beta$-xylanase activity, this enzyme was likely membrane-associated, whereas $\beta$-xylosidase and $\alpha$-L-arabinofuranosidase enzymes can act intracellularly.

The evolution of the microbial community composition over time was assessed for each of the three donors individually, as the PCoA showed that the gut microbiota composition of the three donors differed considerably at species and genus level (Fig. 3). Furthermore, network analysis revealed that only 3 of the 238 , respectively, 19 of the 2503 co-occurrence interactions (edges) were common between the three donors at genus, respectively, species level (Fig. S6). For each donor, a further distinction was observed between the communities from the
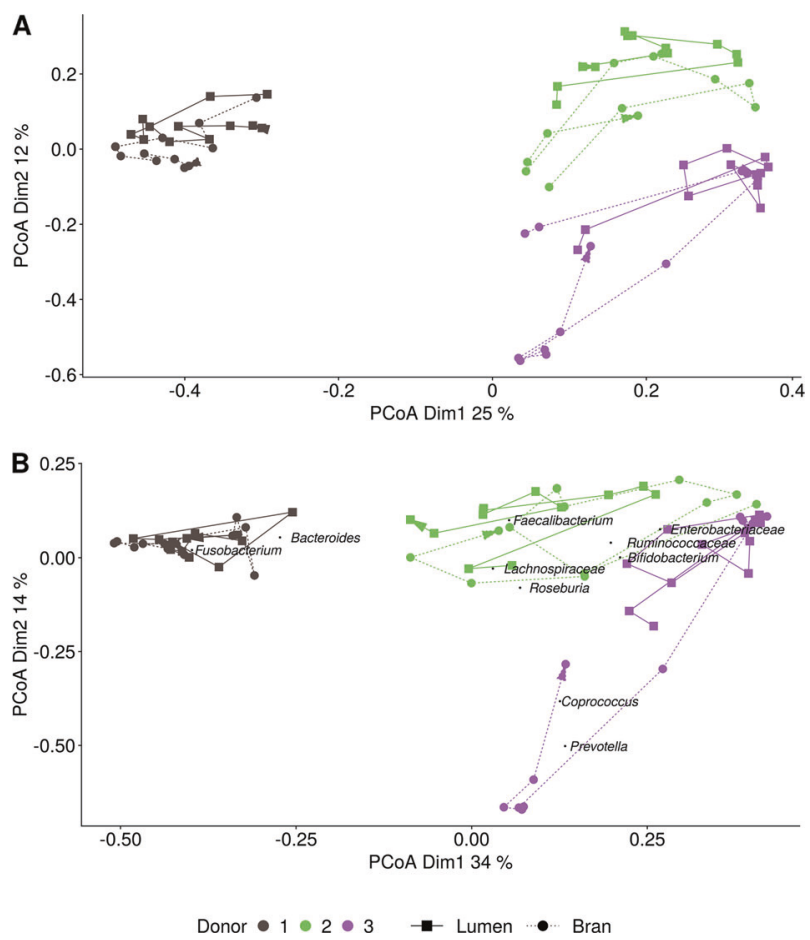

Fig. 3 Principle coordinate analysis $(n=1)$ of the species (a) and genus (b) level luminal (squares) and wheat bran-attached (circles) microbial community composition resulting from the incubation of wheat bran with the faecal material of three different donors for up to $72 \mathrm{~h}$. The abundance-based jaccard dissimilarity matrix was used and weighted average scores of the most abundant genera were a posteriori projected, by means of the wascores function from the package vegan luminal and bran niche, which differed from one another in terms of global community composition and cooccurrence interactions (Figs. 3, S6). For each donor and niche, the variation in the community structure over time was analysed. Both the luminal and bran environment for all three donors displayed a very dynamic microbial community.

In donor 1 , the luminal microbial community radically changed between 2 and $4 \mathrm{~h}$ after inoculation and after a relatively stable period up till $8 \mathrm{~h}$, large fluctuations occurred between 8 and $20 \mathrm{~h}$ after inoculation (Figs. 4a, S7a and S8a). The bran-attached community in donor 1 was less volatile and only displayed a major shift between 2 and $4 \mathrm{~h}$ after inoculation (Figs. 4a, S7a and S8a). In the luminal community, after $2 \mathrm{~h}$, Fusobacterium mortiferum (OTU5) sharply increased in relative abundance at the expense of Bacteroides uniformis (OTU3), Bacteroides stercoris (OTU4) and Faecalibacterium prausnitzii (OTU11, 21) relative abundances (Fig. 5). Between 12 and $16 \mathrm{~h}$, this trend was reversed and the microbial community evolved towards a stable, non-variable proportional composition, dominated by B. uniformis (OTU3) and Firmicutes species belonging to Clostridium cluster XIVa (OTU22, 46), Oscillibacter (OTU31) and after $24 \mathrm{~h}$ also Clostridium cluster IV (OTU53) (Fig. 5). In the bran-attached community, Fusobacterium was gradually replaced by $B$. uniformis (OTU3), B. stercoris (OTU4) and Clostridium xylanolyticum (OTU7). The latter two species showed a clear preference for the bran environment (Fig. 5).

In donor 2, an abrupt change presented itself in the luminal community between 2 and $4 \mathrm{~h}$ after inoculation under the form of a largely increased proportional abundance of Escherichia/Shigella spp. (OTU1) and a drop in Firmicutes species within the genera Faecalibacterium (OTU11,21), Lachnospiraceae (OTU10,50), Ruminococcaceae (OTU153), Clostridiales (OTU7,19,26) and Roseburia (OTU18) (Figs. 4b, 6, S7b and S8b). Shifts in Enterobacteriaceae, comprising Pantoea vagans (OTU15) next to Escherichia-Shigella spp. (OTU1), were also responsible for the large community variation observed in the timespan between 8 and 10, 16 and 20 and 24 and $48 \mathrm{~h}$ (Figs. 4b, 6, S7b and S8b). During these periods Enterobacteriaceae reductions corresponded to elevated levels of B. uniformis (OTU3), Bacteroides ovatus (OTU6), Lachnospiraceae (OTU10,50), Clostridiales (OTU7,26), Ruminococcaceae (OTU153) and Roseburia (OTU18) (Figs. 4b, $6, \mathrm{~S} 7 \mathrm{~b}$, and S8b). The transitions in the luminal community coincided with a big turnover in the bran-attached community (Figs. 4b, 6, S7b, and S8b). The first $16 \mathrm{~h}$, the community composition was dictated by a trade-off between Firmicutes (OTU7,11, 18, 21, 50) and Enterobacteriaceae (OTU1,15) with a slightly increasing Bacteroides share, mainly attributed to B. uniformis (OTU3) 
Fig. 4 Genus level luminal and bran-attached community composition and community similarity between consecutive time points of donor $1(\mathbf{a})$, donor 2 (b) and donor 3 (c) $(n=1)$. The similarity value was calculated by subtracting the abundance based jaccard dissimilarity metric from 1. Similarity values below 0.6 are indicated in red
A
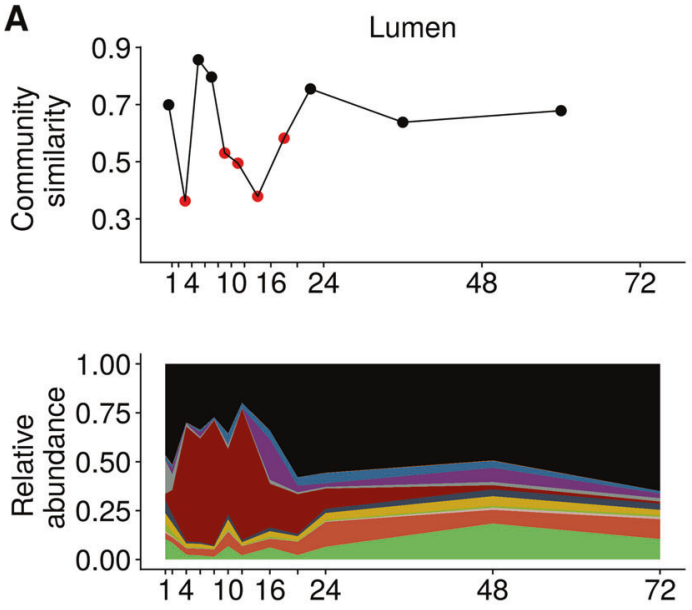

B
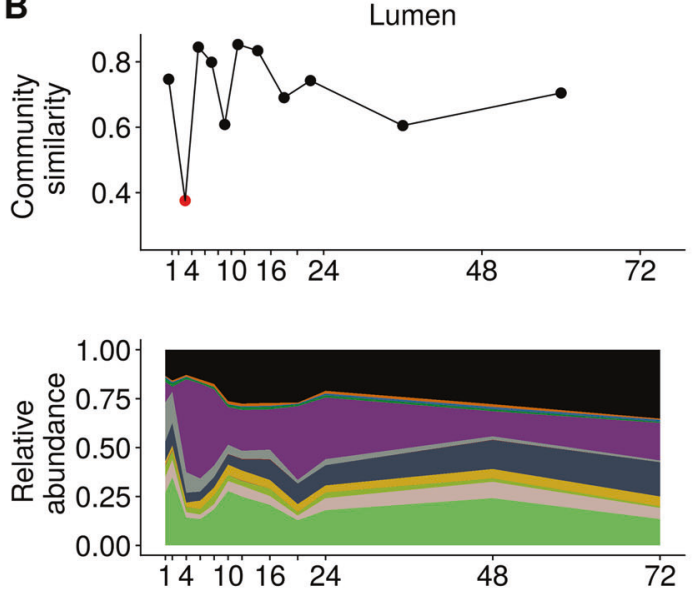

C
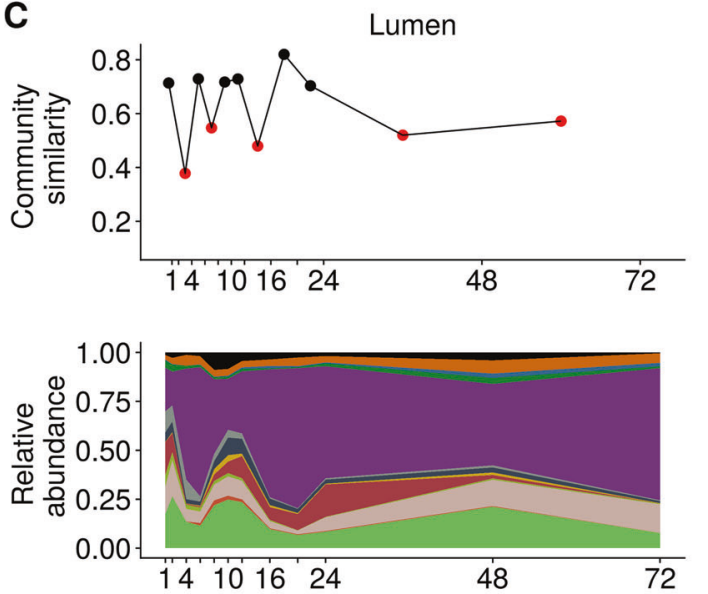

Bacteroides genus Bifidobacterium Clostridium XIVa Coprococcus
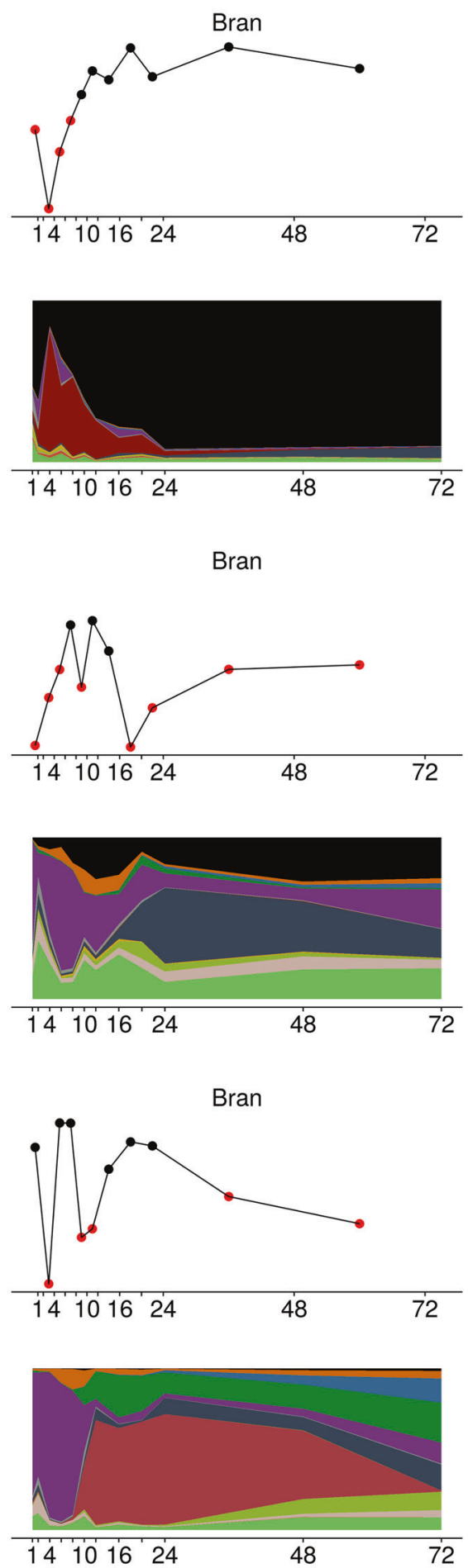

Bran
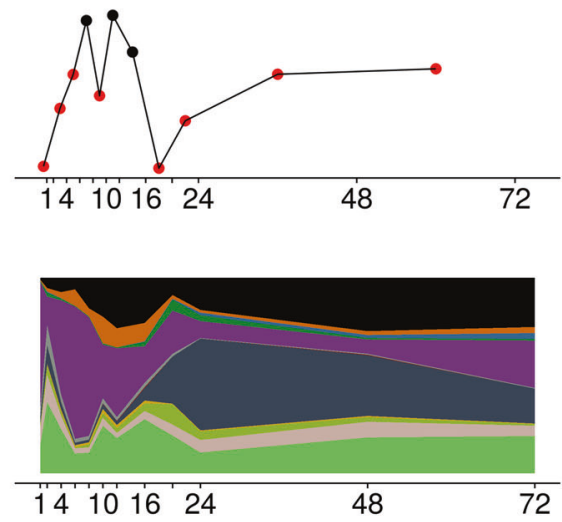

Bran

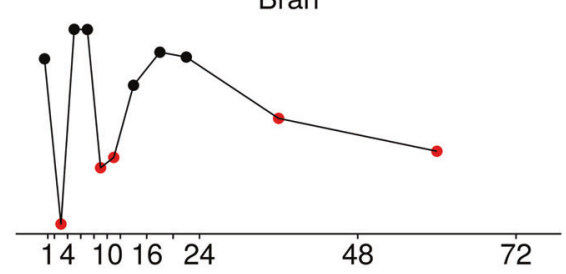

Enterobacteriaceae Faecalibacterium Fusobacterium Lachnospiraceae

\section{Parabacteroides Sutterella \\ Prevotella \\ Roseburia \\ Ruminococcaceae \\ Other}

(Figs. 4b, 6, S7b and S8b). After $20 \mathrm{~h}$, Lachnospiraceae (OTU7,18,19,26,50) and Ruminococcaceae (OTU153) suddenly appeared at high abundance in the bran-attached community and B. ovatus (OTU6) displaced B. uniformis (OTU3) as the dominant bran colonising Bacteroides species (Fig. 6). 

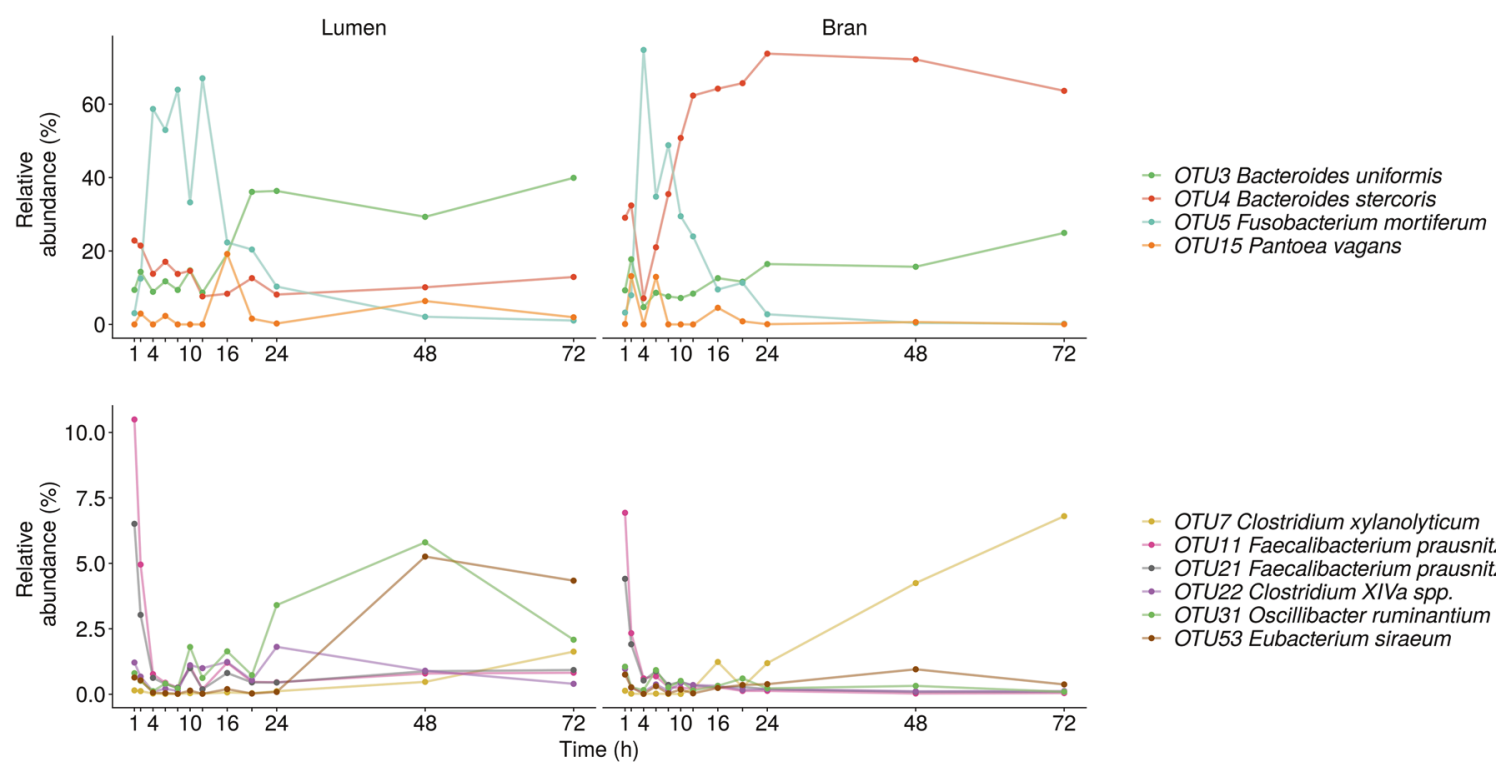

- OTU7 Clostridium xylanolyticum

- OTU11 Faecalibacterium prausnitzi

- OTU21 Faecalibacterium prausnitzi

- OTU22 Clostridium XIVa spp.

- OTU31 Oscillibacter ruminantium

- OTU53 Eubacterium siraeum

Fig. 5 Species level luminal and bran-attached community composition of donor $1(n=1)$
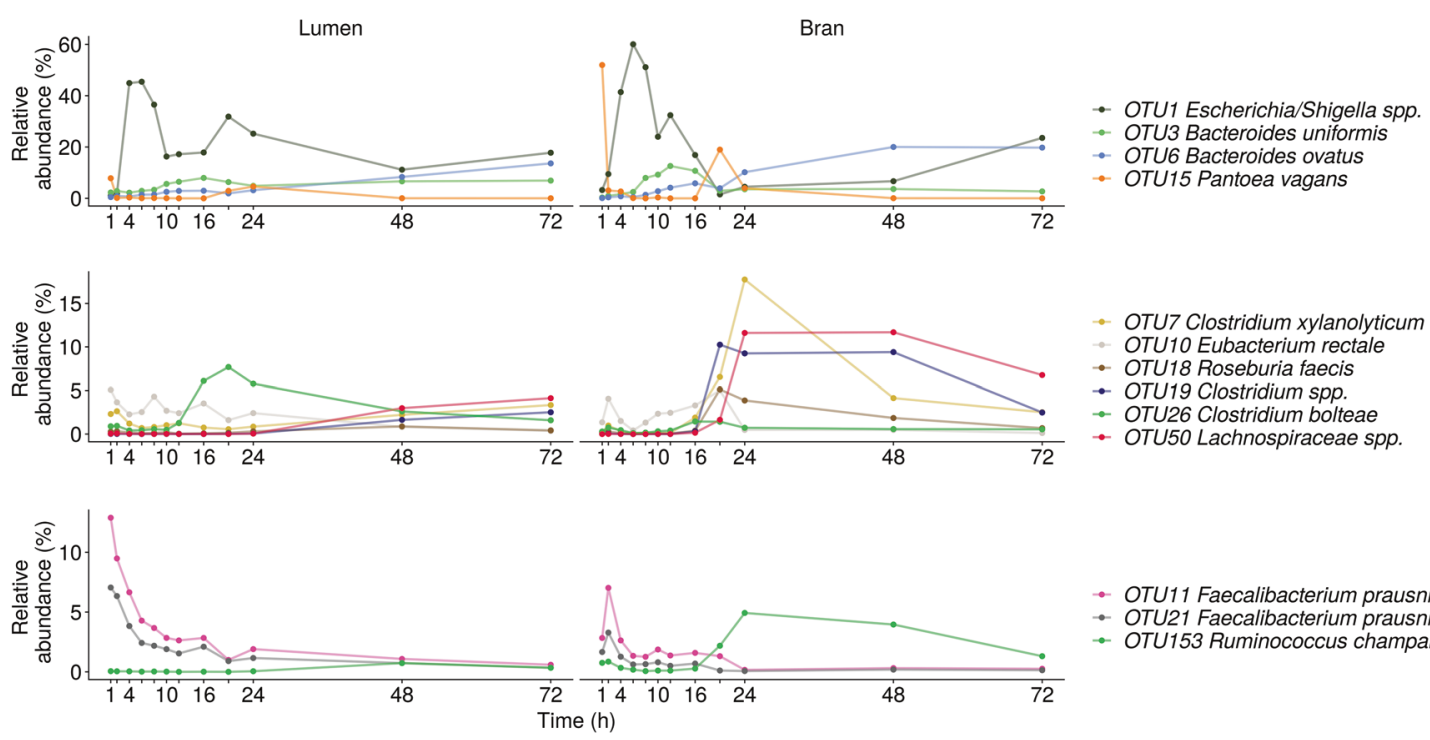

- OTU11 Faecalibacterium prausnitzii

- OTU21 Faecalibacterium prausnitzii

Fig. 6 Species level luminal and bran-attached community composition of donor $2(n=1)$

In donor 3, similar as in donor 2, Proteobacteria and Firmicutes levels alternately increased in the luminal community, causing the peaks in global variability in the timeframes from 2-4, 6-8, and 12-16 h (Fig. S7c). Proteobacteria were represented by Pantoea vagans (OTU15) during the first two hours followed by Escherichia-Shigella spp. (OTU1) (Fig. 7). In the time interval between 8 and 14 $\mathrm{h}$ and after $20 \mathrm{~h}$, Oscillibacter spp. (OTU12) reached a high proportional abundance (Fig. 7). Bacteroidetes abundances remained low in the luminal community throughout time (Fig. S7c). In the bran environment, however, Prevotella copri (OTU8,9) substituted the initially dominating Proteobacteria, after $10 \mathrm{~h}$ (Figs. 4c and 7). Starting at $48 \mathrm{~h}$, diverse Firmicutes members within the Coprococcus (OTU17), Lachnospiraceae (OTU26), Clostridium cluster XIVa (OTU54) and Roseburia (OTU18) genus efficiently colonised the bran (Figs. 4c and 7).

The observed transitions were very abrupt and delimited periods with a more gradually changing community composition (Figs. 4, S7 and S8), which is reflected in the distinctive clustering of time points characterised by a large contrast between the high within-cluster similarity and low betweencluster similarity (Fig. S9). The magnitude of community variation over time, i.e. an abrupt transition versus a more gradual change, could not be explained by the sampling frequency, as indicated by time decay analysis (Fig. S10). The 

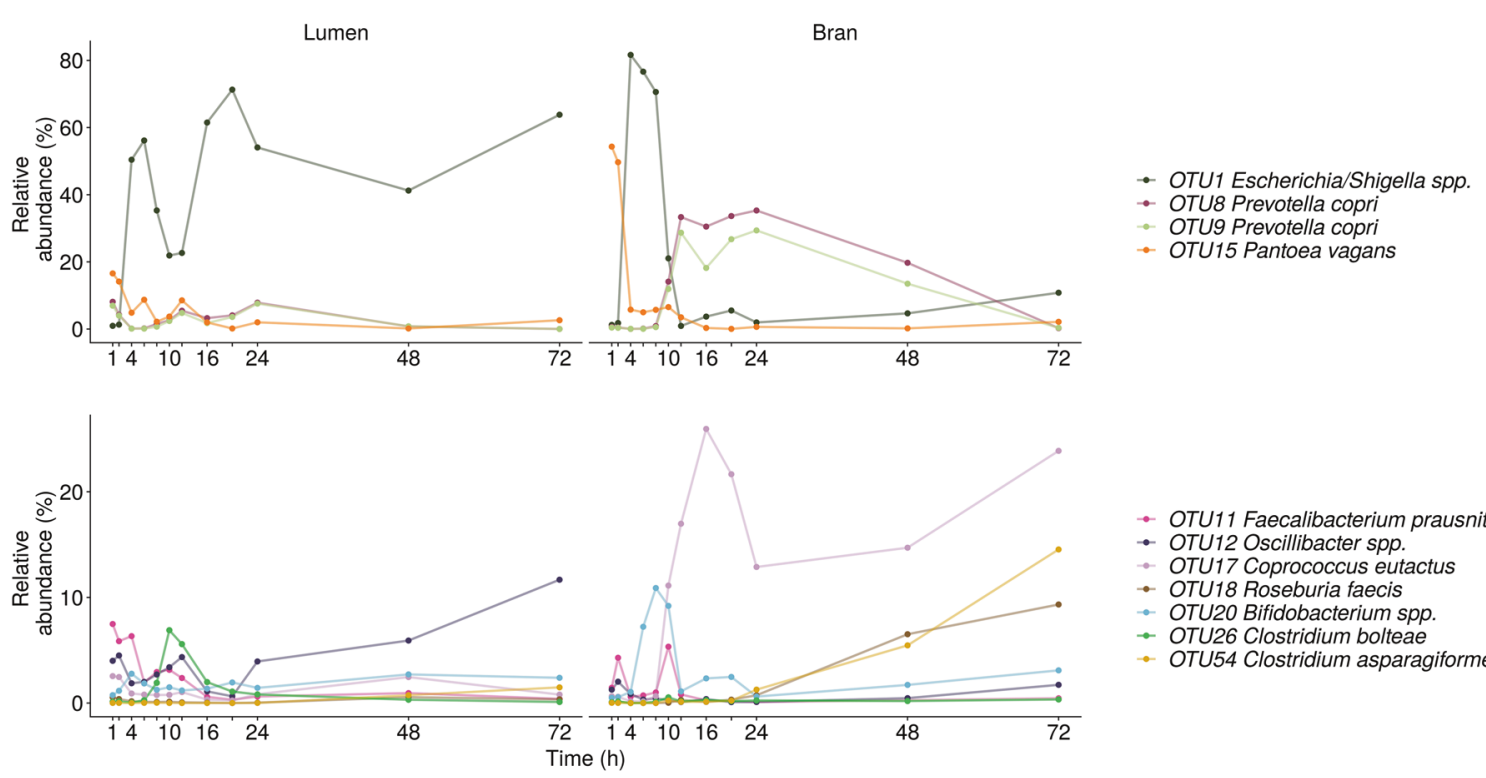

- OTU11 Faecalibacterium prausnitzii

- OTU12 Oscillibacter spp.

- OTU17 Coprococcus eutactus

- OTU18 Roseburia faecis

- OTU20 Bifidobacterium spp.

- OTU26 Clostridium bolteae

OTU54 Clostridium asparagiforme

Fig. 7 Species level luminal and bran-attached community composition of donor $3(n=1)$

community transitions also largely affected the co-occurrence of individual community members, as can be seen from the networks for all donors and niches (Figs. S11-S16).

Network inference confirmed the dominance of Bacteroides and Fusobacterium in donor 1 and Enterobacteriaceae in donors 2 and 3, which translated itself in significant negative co-occurrence interactions with other taxa at genus level both in the bran and luminal community (Figs. S11S16). In contrast, interactions between genera belonging to the Firmicutes phylum were mostly positive. The higher degree of co-existence in the Firmicutes phylum compared to the Bacteroidetes phylum, was also apparent at species level (Fig. 8). The Bacteroidetes community is characterised by the dominance of a single species at all time points.

Finally, to investigate the importance of stochastic effects in the wheat bran colonisation and fermentation process, biological duplicates were analysed. The fermentation products were characterised by a low coefficient of variation of $10 \%$ for acetate, $7 \%$ for propionate, $9 \%$ for butyrate and $21 \%$ for branched SCFA, averaged over all time points $(n=2)$ (Fig. S17a). The amplicon nextgeneration sequencing results from biological replicates at three time points $(2,10$ and $48 \mathrm{~h})$ displayed a higher dispersion, around $50 \%$ at species level (Fig. S17c). This observed dispersion is, however, attributable to both sources of technical and biological variation. Technical variation was estimated by comparing mock community sequencing data $(n=3)$, ruling out biological variation, as the same biological sample was included in triplicate in the sequencing run. The dispersion of the mock community samples was in the same range as those of most biological replicates, suggesting that technical variation accounts to a large extent for the observed dispersion (Fig. S17b, c). Despite the dispersion, replicates clustered together indicating that the wheat bran effects on the microbial community are reproducible (Fig. S17d, e).

\section{Discussion}

Succession is a widely used ecological concept describing a course of changes in species composition over time in response to environmental perturbations [53]. The concept emerged from the observation of plant communities, but has since been explored in many other domains, including microbiology [54, 55]. Well-studied examples of temporal succession in the host-microbe interphase include the colonisation of the infant gut and dental plaque formation $[11,13,56,57]$. In this study, the supplementation of wheat bran as a growth and colonisation substrate resulted in a dynamic microbial succession, characterised by an initial proportional reduction in Faecalibacterium, Lachnospiraceae, Ruminococcaceae, Roseburia and Clostridium cluster XIVa in all three donors, corresponding to a donordependent increase of Fusobacterium and Enterobacteriaceae proportions. These high proportions were in most cases not persistent and gave way to a donor-dependent rise in Bacteroides, Prevotella and Firmicutes over time.

The observed microbial succession was not random, as the microbial community, sampled from separate tubes for each time point, converged to a constant community composition in between shifts. Furthermore, analysis of biological duplicates (for time points 2,10 , and $48 \mathrm{~h}$ ) and a mock community in triplicate indicated that the dispersion, as measured by the coefficient of variation, was mostly imposed by technical variation. Despite the technical noise, 

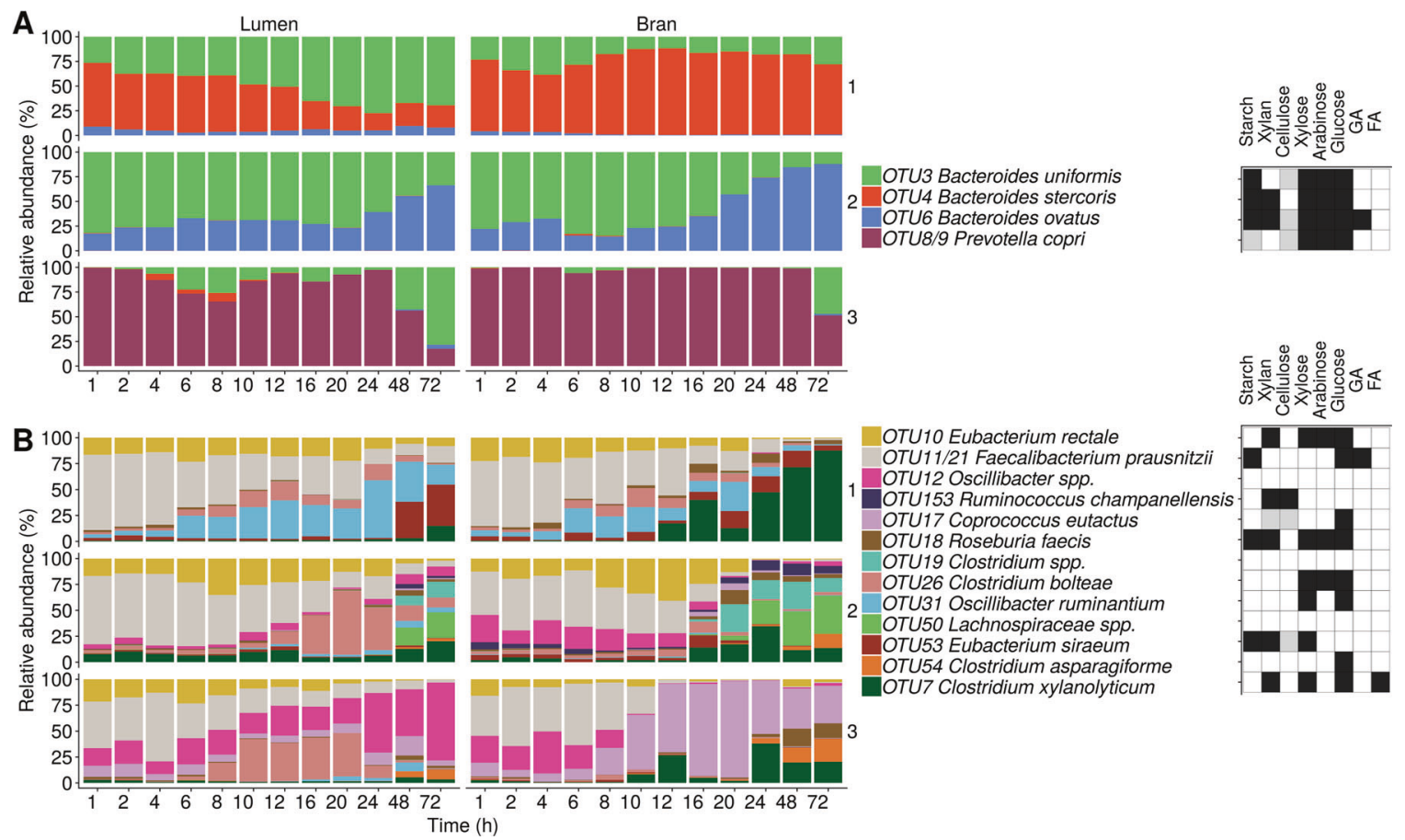

Fig. 8 Luminal and bran-attached community composition of the most abundant species, characteristic for the three donors. Relative abundances of the displayed taxa were normalised and rescaled to $100 \%$ within the Bacteroidetes (a) and Firmicutes (b) phylum $(n=1)$. The experimentally verified (black fill colour) and predicted putative (grey fill colour) capacity of species to degrade wheat bran derived carbohydrates is indicated in a heat map. No fill colour means that there is

biological replicates clustered together indicating that the microbial succession was determined by underlying biological mechanisms. We have explored these possible underlying mechanisms, by reviewing the available literature on the metabolic potency and preferred growth conditions of the most important taxa observed in this study (Supplementary Information; Tables S3-S7). Minimum generation time and oxygen sensitivity were considered as factors that may affect the onset of growth (Supplementary Information; Table S3). $\mathrm{pH}$ optimum and tolerance were examined, as we have demonstrated a pH drop (from 6.8 to 6.2 after $72 \mathrm{~h}$ ) as a consequence of the increased SCFA production over time (Supplementary Information; Table S3). The most essential requirement for the expansion of a population, however, is the metabolic capacity to breakdown a substrate and efficiently assimilate the released monomers and the obtained energy into new biomass. From a comparison of the SCFA profiles in the wheat bran supplemented condition with the control, containing only the carbohydrate-low medium without wheat bran, we have established that nutritional conditions changed as the primary colonisers preferentially consumed the carbohydrate-low medium. Depletion of this medium, consisting of $3 \mathrm{~g} \mathrm{~L}^{-1}$ yeast extract, $1 \mathrm{~g} \mathrm{~L}^{-1}$ peptone and $1 \mathrm{~g} \mathrm{~L}^{-1}$ mucin, prompted the wheat bran colonisation and fermentation by a different set of species. This was no information available that the concerned species is capable of degrading the compounds. Taxa, indicated by spp., which could not be identified to species level, were not considered for the functional annotation. GA glucuronic acid and FA ferulic acid. An extensive functional/physiological characterisation can be found in Supplementary Tables S3-S7

reflected by an increase of the endo-1,4- $\beta$-xylanase activity over time, which was not observed in the control, suggesting that this enzyme activity is inextricably linked to the wheat bran fermentation. This is underscored by the known function of endo-1,4- $\beta$-xylanase in the primary degradation of arabinoxylans, which are a major constituent of wheat bran. Finally, the role of wheat bran as a driver for the observed succession, was further supported by our finding of a different succession pattern on the insoluble wheat bran residue compared to the luminal environment. The driving force behind wheat bran attachment is unclear, but we previously suggested that bacteria deploying the enzymatic capacity to degrade wheat bran could be expected to be located in close proximity of the insoluble substrate [40]. Indeed, Macfarlane et al. [58] reported that the xylanase, $\beta$-xylosidase, $\alpha$ arabinofuranosidase enzyme activity of bacteria desorbed from food particles is significantly higher compared to nonparticulate bacteria [58]. To evaluate if the wheat branattached species in this study have the metabolic capacity to degrade wheat bran carbohydrates, mainly consisting of arabinoxylans and cellulose, a combined literature and database search was performed (described in detail in the Supplementary information; Tables S5-S7). The mechanism of attachment to the insoluble wheat bran substrate are currently unresolved, but could encompass a cellulosome 
enzyme system in case of Ruminococcus champanellensis and common adhesive structures such as pili, fimbriae and extracellular polymeric substances, which were all reviewed in Table S4 [59-62].

In donor $1 F$. mortiferum (OTU5) was predominant in the early stages of incubation in the luminal and branattached microbial community. F. mortiferum is a poorly characterised frequently encountered human gut resident, with established adhesive properties and capable of proteolytic and saccharolytic fermentation (Tables S3 and S7). There is, however, no substantial evidence for the breakdown of complex polysaccharides, such as those present in wheat bran (Tables S5 and S7). In line with this, Fusobacterium proportions started to decline in the bran-attached community after $4 \mathrm{~h}$ and sharply dropped in the luminal community after $16 \mathrm{~h}$, after which the community was dominated by Bacteroides species, which are reported to ferment xylans (Table S7). B. uniformis was most abundant in the luminal community, which is in agreement with the four-fold proportional increase obtained by Duncan et al. [36] after $24 \mathrm{~h}$ of incubation of a faecal sample in the presence of wheat bran. In line with our previous results, $B$. stercoris was more successful in colonising the bran [40].

In donors 2 and 3, Escherichia/Shigella spp. OTU1 was an important primary coloniser. This species reached its maximum relative abundance $4-8 \mathrm{~h}$ after inoculation and sharply decreased in abundance afterwards, except in the luminal community of donor 3. Enterobacteriaceae are fast growing facultative anaerobic opportunistic pathogens, which are phylogenetically closely related (Table S3) [63, 64]. Therefore it comes as no surprise that OTU1 could not be unambiguously classified at species level and showed a similar identity to Escherichia fergusonii, Shigella sonnei, Shigella flexneri and Escherichia coli (Table S2). These species are capable of proteolytic fermentation and produce an extensive CAZyme complement, but are not able to degrade wheat bran arabinoxylans or cellulose (Tables S5-S7).

In donor 2, the decreasing Enterobacteriaceae abundance corresponded to a rise in B. uniformis like in donor 1 , and $B$. ovatus, which was most efficiently colonising the bran surface. B. ovatus is a well-characterised xylanolytic bacteria, capable of growing on wheat bran arabinoxylans and possessing membrane-associated xylan utilisation systems (Xus) directed to the efficient utilisation of soluble xylan through the coordinated cellular attachment, depolymerisation, transport and further intracellular degradation of oligosaccharides [41, 65-67]. This efficient integrated enzyme system also confers an important competitive advantage by avoiding the loss of monosaccharides to other bacteria. Xus have not been extensively experimentally characterised. However, the genes encoding these multienzyme complexes are arranged in polysaccharide utilisation loci (PUL) with a conserved organisation, which can be predicted from genome sequence data [68]. Interestingly, donor 3 was characterised by an enrichment of $P$. copri (OTU8/9) on the bran. This species has also been predicted to carry 6 PULs, containing genes encoding enzymes involved in xylan and cellulose degradation [68] (Table S5 and Fig. 8).

Firmicutes species occurred at low relative abundances during the first 16-20 h, which is reflected in a lagged butyrate production. After this period, possibly favoured by the low $\mathrm{pH}$, the luminal community was characterised by increasing proportions of species related to Oscillibacter ruminantium (OTU31) and Eubacterium siraeum (OTU53) in donor 1, Clostridium bolteae (OTU26) in donor 2 and Oscillibacter spp. (OTU12) in donor 3. The bran was preferentially colonised by $C$. xylanolyticum (OTU7) in donors 1 and 2, Lachnospiraceae spp. (OTU50), Clostridium spp. (OTU19) and R. champanellensis (OTU153) in donor 2 and Coprococcus eutactus (OTU17), Roseburia faecis (OTU18) and Clostridium asparagiforme (OTU54) in donor 3. Most of the identified Firmicutes species were not represented in the CAZy database, reflecting their understudied carbohydrate utilisation capacity and contributing to the misconception that Firmicutes do not play a significant role in carbohydrate utilisation [69]. Recently, it has been suggested that the lower density of genes encoding CAZymes in Firmicutes species is a consequence of a high substrate specificity, determined at species and even strain level [69]. Indeed, in this study, we found species with the reported ability to grow on xylan or xylose comprising $C$. xylanolyticum, $R$. faecis, C. bolteae, O. ruminantium, E. siraeum co-existing with species capable of using cellulose including $R$. champanellensis and C. eutactus (Table S7, Fig. 8). Interestingly, the latter two species are limited in their ability to ferment other carbohydrates and even simple sugars, which was more common among the other species (Table S7). Additionally C. asparigiforme displays $\alpha$ glucuronidase activity, required for the removal of 4-Omethyl-D-glucuronic acid moieties linked to xylose and $C$. xylanolyticum metabolises ferulic acid substituents of arabinose residues in wheat bran arabinoxylans (Table S7 and Fig. 8). R. faecis, moreover, is able to cross-feed on acetate to produce butyrate (Tables S5 and S7) [70, 71]. Butyrate production was related to the proportion of butyrate producing species (Table S7), which was low in donor 1 and reached higher proportions in donors 2 and 3 (R. faecis and C. eutactus).

In summary, during the first stages of incubation Enterobacteriaceae and Fusobacterium species dominated the microbial community. These primary colonisers share many characteristics such as a fast onset of growth at a neutral $\mathrm{pH}$ optimum, oxygen tolerance, adhesive properties, bacteriocin production and a proteolytic and saccharolytic catabolism, mostly directed towards oligosaccharide degradation 
(Tables S3-S7). We therefore hypothesise that these primary colonisers grow well on the supplemented protein rich, carbohydrate-low medium. This is also reflected by their decreased abundance over time, probably due to depletion of the carbohydrate-low medium in combination with acidification. During a second stage of fermentation, characterised by a spike in SCFA production and endo-1,4$\beta$-xylanase enzyme activity, these pioneer species were succeeded by Bacteroidetes and Firmicutes species, which were primarily thriving on the insoluble wheat bran residue. The enrichment of these taxa, possessing the necessary enzymatic capacity to degrade wheat bran, strongly suggests that the succession, at least on the insoluble wheat bran particles, is driven by the nutritional role of wheat bran, acting as a distinct microbial niche. Both Bacteroides and Prevotella species are tailored to breakdown wheat bran by virtue of their extensive glycolytic genomes enriched in xylanolytic genes, whereas the Firmicutes species are less versatile compared to the Bacteroides species and the capacity to degrade xylan, cellulose, starch, ferulic acid or to cross-feed is more divided across species. This high level of specialisation may explain the co-existence of Firmicutes species with a distinct substrate specificity, in contrast to the manifestation of a sole dominant Bacteroidetes species, resulting from their overlapping glycan degrading capacity, which is in line with the inverse relation between Bacteroides and Prevotella observed by others and the positioning of Bacteroides as glycan degrading generalists [1, 72-75].

We realise that the presented in vitro experiment and hypotheses are an oversimplification in the face of the continuous dietary influx and changing host conditions challenging the gut bacteria in vivo. The proposed mechanisms for the microbial succession are speculative given the uncertainty of species level classification based on short-length amplicon reads, the scarce literature data for some of the discussed species and consequent extrapolation of growth characteristics and conditions from close relatives, the automated prediction of growth and metabolic features from genomic information and the underrepresentation of some species in databases. Further research is undoubtedly needed. However, this is to our knowledge the first in-depth study of the short-term dynamics of wheat bran fermentation. It offers a very interesting window into the microbial succession and plausible underlying mechanisms, which are indicative of a niche diversification of species targeting different wheat bran constituents.

Acknowledgements The authors would like to acknowledge the Research Foundation Flanders (FWO, Grant id = IWT130028, title = SBO BRANDING) and the Special Research Fund (BOF) Concerted Research Actions (GOA, BOF12/GOA/008) from the Flemish Government for funding this research. We would like to thank Jolien De Paepe for critically reviewing this manuscript.

\section{Compliance with ethical standards}

Conflict of interest The authors declare that they have no conflict of interest.

Publisher's note Springer Nature remains neutral with regard to jurisdictional claims in published maps and institutional affiliations.

Open Access This article is licensed under a Creative Commons Attribution 4.0 International License, which permits use, sharing, adaptation, distribution and reproduction in any medium or format, as long as you give appropriate credit to the original author(s) and the source, provide a link to the Creative Commons license, and indicate if changes were made. The images or other third party material in this article are included in the article's Creative Commons license, unless indicated otherwise in a credit line to the material. If material is not included in the article's Creative Commons license and your intended use is not permitted by statutory regulation or exceeds the permitted use, you will need to obtain permission directly from the copyright holder. To view a copy of this license, visit http://creativecommons. org/licenses/by/4.0/.

\section{References}

1. Arumugam M, Raes J, Pelletier E, Le Paslier D, Yamada T, Mende DR, et al. Enterotypes of the human gut microbiome. Nature. 2011;473:174-80.

2. Faith JJ, Guruge JL, Charbonneau M, Subramanian S, Seedorf H, Goodman AL, et al. The long-term stability of the human gut microbiota. Science. 2013;341:44-+.

3. Falony G, Joossens M, Vieira-Silva S, Wang J, Darzi Y, Faust K, et al. Population-level analysis of gut microbiome variation. Science. 2016;352:560-4.

4. Li JH, Jia HJ, Cai XH, Zhong HZ, Feng Q, Sunagawa S, et al. An integrated catalog of reference genes in the human gut microbiome. Nat Biotechnol. 2014;32:834-41.

5. Qin JJ, Li RQ, Raes J, Arumugam M, Burgdorf KS, Manichanh C, et al. A human gut microbial gene catalogue established by metagenomic sequencing. Nature. 2010;464:59-U70.

6. The Human Microbiome Project consortium. Structure, function and diversity of the healthy human microbiome. Nature. 2012;486:207-14.

7. Schloissnig S, Arumugam M, Sunagawa S, Mitreva M, Tap J, Zhu A, et al. Genomic variation landscape of the human gut microbiome. Nature. 2013;493:45-50.

8. Walter J, Ley R. The human gut microbiome: ecology and recent evolutionary changes. Annu Rev Microbiol. 2011;65:411-29.

9. Caporaso JG, Lauber CL, Costello EK, Berg-Lyons D, Gonzalez A, Stombaugh J, et al. Moving pictures of the human microbiome. Genome Biol. 2011;12:R50.

10. Faust K, Lahti L, Gonze D, de Vos WM, Raes J. Metagenomics meets time series analysis: unraveling microbial community dynamics. Curr Opin Microbiol. 2015;25:56-66.

11. Koenig JE, Spor A, Scalfone N, Fricker AD, Stombaugh J, Knight $\mathrm{R}$, et al. Succession of microbial consortia in the developing infant gut microbiome. Proc Natl Acad Sci USA. 2011;108:4578-85.

12. Pop M. We are what we eat: how the diet of infants affects their gut microbiome. Genome Biol. 2012;13:152.

13. Vallès Y, Artacho A, Pascual-García A, Ferrús ML, Gosalbes MJ, Abellán JJ, et al. Microbial succession in the gut: directional trends of taxonomic and functional change in a Birth Cohort of Spanish Infants. Plos Genet. 2014;10:e1004406.

14. David LA, Materna AC, Friedman J, Campos-Baptista MI, Blackburn MC, Perrotta A, et al. Host lifestyle affects human microbiota on daily timescales. Genome Biol. 2014;15:R89. 
15. DiGiulio DB, Callahan BJ, McMurdie PJ, Costello EK, Lyell DJ, Robaczewska A, et al. Temporal and spatial variation of the human microbiota during pregnancy. Proc Natl Acad Sci USA. 2015;112:11060-5.

16. Rajilic-Stojanovic M, Heilig HGHJ, Tims S, Zoetendal EG, de Vos WM. Long-term monitoring of the human intestinal microbiota composition. Environ Microbiol. 2013;15:1146-59.

17. Lawley TD, Walker AW. Intestinal colonization resistance. Immunology. 2013;138:1-11.

18. $\mathrm{Xu} \mathrm{ZJ}$, Knight R. Dietary effects on human gut microbiome diversity. Br J Nutr. 2015;113:S1-5.

19. Zaura E, Brandt BW, de Mattos MJT, Buijs MJ, Caspers MPM, Rashid MU, et al. Same exposure but two radically different responses to antibiotics: resilience of the salivary microbiome versus long-term microbial shifts in feces. Mbio. 2015;6: e01693-15.

20. Conlon MA, Bird AR. The impact of diet and lifestyle on gut microbiota and human health. Nutrients. 2014;7:17-44.

21. David LA, Maurice CF, Carmody RN, Gootenberg DB, Button JE, Wolfe BE, et al. Diet rapidly and reproducibly alters the human gut microbiome. Nature. 2014;505:559-63.

22. Flint HJ, Duncan SH, Scott KP, Louis P. Interactions and competition within the microbial community of the human colon: links between diet and health. Environ Microbiol. 2007;9:1101-11.

23. Wu GD, Chen J, Hoffmann C, Bittinger K, Chen YY, Keilbaugh $\mathrm{SA}$, et al. Linking long-term dietary patterns with gut microbial enterotypes. Science. 2011;334:105-8.

24. Costabile A, Klinder A, Fava F, Napolitano A, Foglian V, Leonard $\mathrm{C}$, et al. Whole-grain wheat breakfast cereal has a prebiotic effect on the human gut microbiota: a double-blind, placebocontrolled, crossover study. Br J Nutr. 2008;99:110-20.

25. Costabile A, Kolida S, Klinder A, Gietl E, Bauerlein M, Frohberg $\mathrm{C}$, et al. A double-blind, placebo-controlled, cross-over study to establish the bifidogenic effect of a very-long-chain inulin extracted from globe artichoke (Cynara scolymus) in healthy human subjects. Br J Nutr. 2010;104:1007-17.

26. Damen B, Verspreet J, Pollet A, Broekaert WF, Delcour JA, Courtin CM. Prebiotic effects and intestinal fermentation of cereal arabinoxylans and arabinoxylan oligosaccharides in rats depend strongly on their structural properties and joint presence. Mol Nutr Food Res. 2011;55:1862-74.

27. Kolida S, Meyer D, Gibson GR. A double-blind placebo-controlled study to establish the bifidogenic dose of inulin in healthy humans. Eur J Clin Nutr. 2007;61:1189-95.

28. Ramirez-Farias C, Slezak K, Fuller Z, Duncan A, Holtrop G, Louis P. Effect of inulin on the human gut microbiota: stimulation of Bifidobacterium adolescentis and Faecalibacterium prausnitzii. Br J Nutr. 2009;101:541-50.

29. Bouhnik Y, Raskine L, Simoneau G, Vicaut E, Neut C, Flourie B, et al. The capacity of nondigestible carbohydrates to stimulate fecal bifidobacteria in healthy humans: a double-blind, randomized, placebo-controlled, parallel-group, dose-response relation study. Am J Clin Nutr. 2004;80:1658-64.

30. Walton GE, Lu CY, Trogh I, Arnaut F, Gibson GR. A randomised, double-blind, placebo controlled cross-over study to determine the gastrointestinal effects of consumption of arabinoxylan-oligosaccharides enriched bread in healthy volunteers. Nutr J. 2012;11:36.

31. Walker AW, Ince J, Duncan SH, Webster LM, Holtrop G, Ze XL, et al. Dominant and diet-responsive groups of bacteria within the human colonic microbiota. ISME J. 2011;5:220-30.

32. Aguirre M, Eck A, Koenen ME, Savelkoul PH, Budding AE, Venema K. Diet drives quick changes in the metabolic activity and composition of human gut microbiota in a validated in vitro gut model. Res Microbiol. 2016;167:114-25.
33. Cummings JH, Danone I. The large intestine in nutrition and disease. Institut Danone; Brussels, Belgium, 1997.

34. Venema K, van den Abbeele P. Experimental models of the gut microbiome. Best Pract Res Clin Gastroenterol. 2013;27:115-26.

35. Yao CK, Gibson PR, Shepherd SJ. Design of clinical trials evaluating dietary interventions in patients with functional gastrointestinal disorders. Am J Gastroenterol. 2013;108:748-58.

36. Duncan SH, Russell WR, Quartieri A, Rossi M, Parkhill J, Walker $\mathrm{AW}$, et al. Wheat bran promotes enrichment within the human colonic microbiota of butyrate-producing bacteria that release ferulic acid. Environ Microbiol. 2016;18:2214-25.

37. Hemdane S, Jacobs PJ, Dornez E, Verspreet J, Delcour JA, Courtin CM. Wheat (Triticum aestivum L.) bran in bread making: a critical review. Compr Rev Food Sci Food Saf. 2016;15:28-42.

38. Jones JM. Dietary fiber future directions: integrating new definitions and findings to inform nutrition research and communication. Adv Nutr. 2013;4:8-15.

39. Leitch ECM, Walker AW, Duncan SH, Holtrop G, Flint HJ. Selective colonization of insoluble substrates by human faecal bacteria. Environ Microbiol. 2007;9:667-79.

40. De Paepe K, Kerckhof F-M, Verspreet J, Courtin CM, Van de Wiele T. Inter-individual differences determine the outcome of wheat bran colonization by the human gut microbiome. Environ Microbiol. 2017;19:3251-67.

41. Dodd D, Mackie RI, Cann IKO. Xylan degradation, a metabolic property shared by rumen and human colonic Bacteroidetes. Mol Microbiol. 2011;79:292-304.

42. Flint HJ, Scott KP, Duncan SH, Louis P, Forano E. Microbial degradation of complex carbohydrates in the gut. Gut Microbes. 2012;3:289-306.

43. Biely P. Microbial xylanolytic systems. Trends Biotechnol. 1985;3:286-90.

44. De Paepe K, Verspreet J, Rezaei MN, Martinez SH, Meysman F, Van de Walle D, et al. Modification of wheat bran particle size and tissue composition affects colonisation and metabolism by human faecal microbiota. Food Funct. 2019;10:379-96.

45. Minekus M, Alminger M, Alvito P, Ballance S, Bohn T, Bourlieu $\mathrm{C}$, et al. A standardised static in vitro digestion method suitable for food - an international consensus. Food Funct. 2014;5:1113-24.

46. Klindworth A, Pruesse E, Schweer T, Peplies J, Quast C, Horn M, et al. Evaluation of general $16 \mathrm{~S}$ ribosomal RNA gene PCR primers for classical and next-generation sequencing-based diversity studies. Nucleic Acids Res. 2013;41:e1.

47. Kozich JJ, Westcott SL, Baxter NT, Highlander SK, Schloss PD. Development of a dual-index sequencing strategy and curation pipeline for analyzing amplicon sequence data on the MiSeq illumina sequencing platform. Appl Environ Micro. 2013;79: 5112-20.

48. R Core Team. R: a language and environment for statistical computing. Vienna, Austria: R Foundation for Statistical Computing; 2016. https://www.R-project.org/.

49. McMurdie PJ, Holmes S. Waste not, want not: why rarefying microbiome data is inadmissible. Plos Comput Biol. 2014;10: e1003531.

50. Altschul SF, Gish W, Miller W, Myers EW, Lipman DJ. Basic local alignment search tool. J Mol Biol. 1990;215:403-10.

51. Wang Q, Garrity GM, Tiedje JM, Cole JR. Naive Bayesian classifier for rapid assignment of rRNA sequences into the new bacterial taxonomy. Appl Environ Microbiol. 2007;73:5261-7.

52. Cole JR, Wang Q, Fish JA, Chai BL, McGarrell DM, Sun YN, et al. Ribosomal Database Project: data and tools for high throughput rRNA analysis. Nucleic Acids Res. 2014;42:D633-42.

53. Connell JH, Slatyer RO. Mechanisms of succession in natural communities and their role in community stability and organization. Am Nat. 1977;111:1119-44. 
54. Faust K, Raes J. Microbial interactions: from networks to models. Nat Rev Microbiol. 2012;10:538-50.

55. Jiao S, Chen WM, Wang ET, Wang JM, Liu ZS, Li YN, et al. Microbial succession in response to pollutants in batchenrichment culture. Sci Rep. 2016;6:21791.

56. Faust K, Sathirapongsasuti JF, Izard J, Segata N, Gevers D, Raes $\mathrm{J}$, et al. Microbial co-occurrence relationships in the human microbiome. Plos Comput Biol. 2012;8:e1002606.

57. Bradshaw DJ, Marsh PD, Watson GK, Allison C. Role of Fusobacterium nucleatum and coaggregation in anaerobe survival in planktonic and biofilm oral microbial communities during aeration. Infect Immun. 1998;66:4729-32.

58. Macfarlane S, McBain AJ, Macfarlane GT. Consequences of biofilm and sessile growth in the large intestine. Adv Dent Res. 1997;11:59-68

59. Bäckhed F, Ley RE, Sonnenburg JL, Peterson DA, Gordon JI. Host-bacterial mutualism in the human intestine. Science. 2005;307:1915-20.

60. Morais S, Ben David Y, Bensoussan L, Duncan SH, Koropatkin NM, Martens EC, et al. Enzymatic profiling of cellulosomal enzymes from the human gut bacterium, Ruminococcus champanellensis, reveals a fine-tuned system for cohesin-dockerin recognition. Environ Microbiol. 2016;18:542-56.

61. Van Wey AS, Cookson AL, Roy NC, McNabb WC, Soboleva TK, Shorten PR. Bacterial biofilms associated with food particles in the human large bowel. Mol Nutr Food Res. 2011;55:969-78.

62. Ventura M, Turroni F, Motherway MO, MacSharry J, van Sinderen D. Host-microbe interactions that facilitate gut colonization by commensal bifidobacteria. Trends Microbiol. 2012;20:467-76.

63. Greub G. Culturomics: a new approach to study the human microbiome. Clin Microbiol Infect. 2012;18:1157-9.

64. Rivera-Chavez F, Lopez CA, Baumler AJ. Oxygen as a driver of gut dysbiosis. Free Radic Bio Med. 2017;105:93-101.
65. McNulty NP, Wu M, Erickson AR, Pan CL, Erickson BK, Martens $\mathrm{EC}$, et al. Effects of diet on resource utilization by a model human gut microbiota containing bacteroides cellulosilyticus $\mathrm{WH} 2$, a symbiont with an extensive glycobiome. Plos Biol. 2013;11:e1001637.

66. Rogowski A, Briggs JA, Mortimer JC, Tryfona T, Terrapon N, Lowe EC, et al. Glycan complexity dictates microbial resource allocation in the large intestine. Nat Commun. 2016;7:10705.

67. White BA, Lamed R, Bayer EA, Flint HJ. Biomass utilization by gut microbiomes. Annu Rev Microbiol. 2014;68:279-96.

68. Terrapon N, Lombard V, Gilbert HJ, Henrissat B. Automatic prediction of polysaccharide utilization loci in Bacteroidetes species. Bioinformatics. 2015;31:647-55.

69. Sheridan PO, Martin JC, Lawley TD, Browne HP, Harris HM, Bernalier-Donadille A, et al. Polysaccharide utilization loci and nutritional specialization in a dominant group of butyrate-producing human colonic Firmicutes. Micro Genom. 2016;2:e000043.

70. Duncan SH, Holtrop G, Lobley GE, Calder AG, Stewart CS, Flint HJ. Contribution of acetate to butyrate formation by human faecal bacteria. Br J Nutr. 2004;91:915-23.

71. Louis P, Flint HJ. Diversity, metabolism and microbial ecology of butyrate-producing bacteria from the human large intestine. Fems Microbiol Lett. 2009;294:1-8.

72. El Kaoutari A, Armougom F, Gordon JI, Raoult D, Henrissat B. The abundance and variety of carbohydrate-active enzymes in the human gut microbiota. Nat Rev Microbiol. 2013;11:497-504.

73. Gorvitovskaia A, Holmes SP, Huse SM. Interpreting prevotella and bacteroides as biomarkers of diet and lifestyle. Microbiome. 2016;4:15.

74. Berry D. The emerging view of Firmicutes as key fibre degraders in the human gut. Environ Microbiol. 2016;18:5305-5.

75. Koropatkin NM, Cameron EA, Martens EC. How glycan metabolism shapes the human gut microbiota. Nat Rev Microbiol. 2012;10:323-35 